\title{
Lagrangian statistics of turbulent channel flow at Reт $=950$ calculated with direct numerical simulation and Langevin models
}

\section{Citation for published version (APA):}

Kuerten, J. G. M., \& Brouwers, J. J. H. (2013). Lagrangian statistics of turbulent channel flow at Ret $=950$ calculated with direct numerical simulation and Langevin models. Physics of Fluids, 25(10), 105108-1/12. [105108]. https://doi.org/10.1063/1.4824795

DOI:

$10.1063 / 1.4824795$

Document status and date:

Published: 01/01/2013

\section{Document Version:}

Publisher's PDF, also known as Version of Record (includes final page, issue and volume numbers)

\section{Please check the document version of this publication:}

- A submitted manuscript is the version of the article upon submission and before peer-review. There can be important differences between the submitted version and the official published version of record. People interested in the research are advised to contact the author for the final version of the publication, or visit the DOI to the publisher's website.

- The final author version and the galley proof are versions of the publication after peer review.

- The final published version features the final layout of the paper including the volume, issue and page numbers.

Link to publication

\footnotetext{
General rights

- You may freely distribute the URL identifying the publication in the public portal. follow below link for the End User Agreement:

www.tue.nl/taverne

\section{Take down policy}

If you believe that this document breaches copyright please contact us at:

openaccess@tue.nl

providing details and we will investigate your claim.
}

Copyright and moral rights for the publications made accessible in the public portal are retained by the authors and/or other copyright owners and it is a condition of accessing publications that users recognise and abide by the legal requirements associated with these rights.

- Users may download and print one copy of any publication from the public portal for the purpose of private study or research.

- You may not further distribute the material or use it for any profit-making activity or commercial gain

If the publication is distributed under the terms of Article $25 \mathrm{fa}$ of the Dutch Copyright Act, indicated by the "Taverne" license above, please 


\title{
Lagrangian statistics of turbulent channel flow at $\mathbf{R e}_{\tau}=950$ calculated with direct numerical simulation and Langevin models
}

\author{
J. G. M. Kuerten ${ }^{\text {a) }}$ and J. J. H. Brouwers ${ }^{\text {b) }}$ \\ Department of Mechanical Engineering, Eindhoven University of Technology, P.O. Box 513, \\ NL-5600 MB Eindhoven, The Netherlands
}

(Received 6 June 2013; accepted 20 September 2013; published online 15 October 2013)

\begin{abstract}
We present results of Lagrangian statistical quantities for direct numerical simulation (DNS) of turbulent channel flow at Reynolds number $\mathrm{Re}_{\tau}=950$ based on shear velocity and channel half-height. Attention is focused on time correlations of fluid particle velocity and on the wall-normal diffusivity as a function of the wall-normal distance. Away from the wall region the DNS results compare favorably with the results of recent statistical models based on Kolmogorov theory and Onsager symmetry relations. It is found that a value for the Kolmogorov constant of $C_{0}=6$ gives optimal agreement between DNS results and results of the statistical models for all quantities considered. @ 2013 AIP Publishing LLC. [http://dx.doi.org/10.1063/1.4824795]
\end{abstract}

\section{INTRODUCTION}

One of the unsolved problems of physics is a statistical description of turbulent flow and in particular a description of particle dispersion in turbulent flow. Conventionally, the approach adopted for the description of turbulent dispersion is a diffusion model, ${ }^{1}$ but this can only rigorously be derived for the case of homogeneous and stationary turbulence. ${ }^{1-3}$ For the statistical description of particle dispersion in inhomogeneous turbulence the approach based on the diffusion model is more heuristic and involves dimensional arguments and fitting unknown parameters to experimental results.

A more promising approach for dispersion modeling in inhomogeneous turbulent flow is based on a Langevin model for the velocity of a passive particle. This approach is consistent with Kolmogorov theory for the asymptotic structure of turbulent flow at large Reynolds number. ${ }^{1}$ In this approach the most important issue is the specification of the damping function in the Langevin equation. For homogeneous isotropic turbulence this issue has rigorously been solved. ${ }^{4,5}$ However, for inhomogeneous turbulence a unique determination of the damping function has remained an unsolved issue until recently. ${ }^{4,6}$

Recently, a way out of the non-uniqueness problem has been proposed, which is based on an expansion in the reciprocal of the universal Kolmogorov constant $C_{0}$ and invokes Onsager symmetry. ${ }^{7-9}$ This expansion is valid if the Kolmogorov constant is sufficiently large, which requires a rather high Reynolds number. Moreover, the Langevin model disregards the effects of viscosity at small time scales, which are appreciable at limited values of the Reynolds number. In order to assess the validity of the recently developed model, Lagrangian statistical quantities for inhomogeneous turbulent flow at large Reynolds number are required.

During the latest decades the Lagrangian approach to fluid mechanics has received increased attention. The difficulty in the Lagrangian approach is the establishment of fluid mechanical properties associated with the moving particles, which is in contrast with the description in a fixed Eulerian

\footnotetext{
a) Also at Faculty EEMCS, University of Twente, P.O. Box 217, 7500 AE Enschede, The Netherlands. Electronic mail: j.g.m.kuerten@tue.nl.

b) Electronic mail: j.j.h.brouwers@tue.nl
} 
frame. The situation has improved by the availability of accurate experimental and numerical particle tracking methods. ${ }^{10}$ Nowadays, direct numerical simulation (DNS) of turbulent channel flow has been performed at Reynolds numbers based on shear velocity and half the channel height larger than 2000. ${ }^{11}$ However, calculation of Lagrangian statistics has not been reported yet at such high Reynolds numbers.

In this paper, we present new results of DNS with Lagrangian particle tracking in turbulent channel flow at Reynolds number $\mathrm{Re}_{\tau}=950$ based on shear velocity and channel half-height. ${ }^{12}$ The results are compared with predictions of the recently developed stochastic model. ${ }^{7}$ We will compare Lagrangian velocity correlation functions and also diffusion coefficients, which are obtained from the statistical properties of a passive scalar coupled to the DNS of the fluid flow.

In Sec. II we will present the governing equations and numerical method applied in the DNS and for the calculation of the trajectories of fluid particles. Sections III and IV contain a description of the statistical models applied in this paper, together with the numerical method to solve these models. Results are shown in Secs. V and VI and conclusions stated in Sec. VII.

\section{DIRECT NUMERICAL SIMULATION}

In this paper, we consider turbulent incompressible flow in a plane channel, i.e., between two infinite flat plates. The governing equations are the continuity and the Navier-Stokes equation:

$$
\begin{gathered}
\nabla \cdot \mathbf{u}=0, \\
\frac{\partial \mathbf{u}}{\partial t}+\frac{1}{\rho} \nabla p=\mathbf{f}-\boldsymbol{\omega} \times \mathbf{u}+v \nabla^{2} \mathbf{u} .
\end{gathered}
$$

In these equations $\rho, v$, and $\mathbf{u}$ denote mass density, kinematic viscosity, and velocity of the fluid, $p$ total pressure, $\omega$ vorticity, and $t$ time. The equations are made non-dimensional using the mass density, half the channel height, $H$, and the friction velocity, $u_{\tau}=\sqrt{\tau / \rho}$ with $\tau$ the shear stress, as mass density, length, and velocity scale, so that $\operatorname{Re}_{\tau}$ is the friction Reynolds number given by

$$
\operatorname{Re}_{\tau}=\frac{H u_{\tau}}{v} .
$$

The friction Reynolds number of the flow is kept fixed by prescribing the mean driving force per unit mass $\mathbf{f}$ in the streamwise direction parallel to the plates. This implies that the bulk Reynolds number, based on the bulk velocity, fluctuates in time around a mean value. The velocity satisfies no-slip conditions at the two plates. In the other two directions periodic boundary conditions are applied for velocity and pressure. The length of the domain equals $2 \pi H$ in streamwise direction and $\pi H$ in the spanwise direction. Throughout the paper we will use $x_{1}, x_{2}$, and $x_{3}$ for the streamwise, wall-normal and spanwise coordinates and directions.

The periodic boundary conditions in two coordinate directions make the use of a pseudo-spectral method very convenient. In these two periodic directions a Fourier-Galerkin approach is chosen, whereas the wall-normal direction is treated by a Chebyshev-tau method. The incompressibility constraint is satisfied by using the wall-normal component of the vorticity vector and the Laplacian of the wall-normal velocity component as dependent variables, instead of the three velocity components. Hence, the spatial discretization of the problem for the gas velocity closely follows the method by Kim et al. ${ }^{13}$ Nonlinear terms are calculated in physical space by fast Fourier transform (FFT) with application of the $3 / 2$ rule in both periodic directions. For integration in time a combination of a second-order accurate three-stage Runge-Kutta method and the implicit Crank-Nicolson method is chosen according to Spalart et al. ${ }^{14}$ In this way the nonlinear terms are treated in an explicit way, whereas the linear terms are treated implicitly.

Simulations are performed at a friction Reynolds number equal to $\operatorname{Re}_{\tau}=950$, which corresponds to a bulk Reynolds number of approximately 18 900. The numbers of Fourier modes in the two periodic directions equal 768, whereas 385 Chebyshev polynomials are used in the wall-normal direction. The resolution in the wall-normal direction is equal to the one applied by Hoyas and Jiménez. ${ }^{15}$ The grid spacing equals $7.8 v u_{\tau}^{-1}$ in streamwise direction and $3.9 v u_{\tau}^{-1}$ in spanwise 


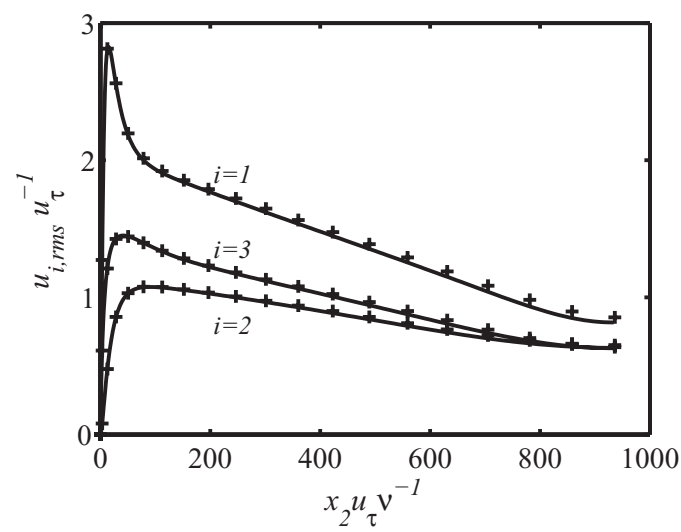

FIG. 1. Root-mean square of the three velocity components as functions of the wall-normal coordinate in wall units; from top to bottom: streamwise, spanwise, and wall-normal component; solid lines: Hoyas and Jiménez; ${ }^{15}+$ : present results.

direction. This is a higher resolution than used by Hoyas and Jiménez, ${ }^{15}$ but our computational domain is much smaller. The time step used in the simulation equals $0.095 v u_{\tau}^{-2}$.

The simulations reported in this paper start from an initial solution in the statistically steady turbulent state. First and second moments of the velocity in this state agree well with results by Hoyas and Jiménez, ${ }^{15}$ as can be seen in Fig. 1, where the root-mean square of the three velocity components is compared. The results shown here are averaged over a time span $5.7 \times 10^{4} v u_{\tau}^{-2}$. Also at lower frictional Reynolds numbers the numerical method has been validated by comparison with results from literature. ${ }^{16}$

In order to calculate Lagrangian velocity correlation functions passive tracers are added to the flow. They obey the equation

$$
\frac{d \mathbf{x}}{d t}=\mathbf{u}(\mathbf{x}(t), t)
$$

where $\mathbf{x}$ is the position of a tracer particle. The equation for each particle is solved numerically with the same explicit second-order accurate Runge-Kutta method as applied to the solution of the Navier-Stokes equation. In order to evaluate the right-hand side of (4) the fluid velocity needs to be interpolated to the position of the tracer. We apply tri-linear interpolation, but use the velocity field after Fourier transformation to real space in which the number of grid points in the two periodic directions is increased by a factor of $3 / 2$. Although higher-order interpolation methods yield a higher accuracy for single particle results, we checked that the accuracy of statistical particle results is not significantly affected by the low order interpolation. If a particle leaves the domain through one of the periodic boundaries, it is reinserted at the opposite boundary. Due to the behavior of the wall-normal velocity component close to a wall, collisions of tracer particles with a wall do not occur.

Lagrangian statistical quantities depend on the wall-normal coordinate, which is the only inhomogeneous direction in channel flow. In order to determine these quantities as functions of the wall-normal coordinates we insert particles at 24 different values of $x_{2}$. For every value of $x_{2} 100$ particles are inserted, homogeneously distributed over the two periodic directions and on both sides of the channel center plane. After every time step the position and velocity of each particle is written to file for later evaluation of the statistical quantities. After 2000 time steps (which corresponds to $t=190 \nu u_{\tau}^{-2}$ ) the velocity correlations have decreased sufficiently and new tracer particles are inserted into the flow. In 2000 time steps a particle traveling with the bulk fluid velocity covers a distance of $63 \%$ of the channel length. In this way 300 independent results, each with 100 particles for 24 different $x_{2}$ values have been collected.

In the post-processing step we calculate Lagrangian averages and velocity correlation functions. The velocity correlation functions $\left\langle v_{i}(t) v_{j}\left(t_{0}\right)\right\rangle$, where $\mathbf{v}(t)$ is the velocity of a tracer particle at time $t$ minus the Eulerian mean velocity at the position of the particle, only depend on the time difference 


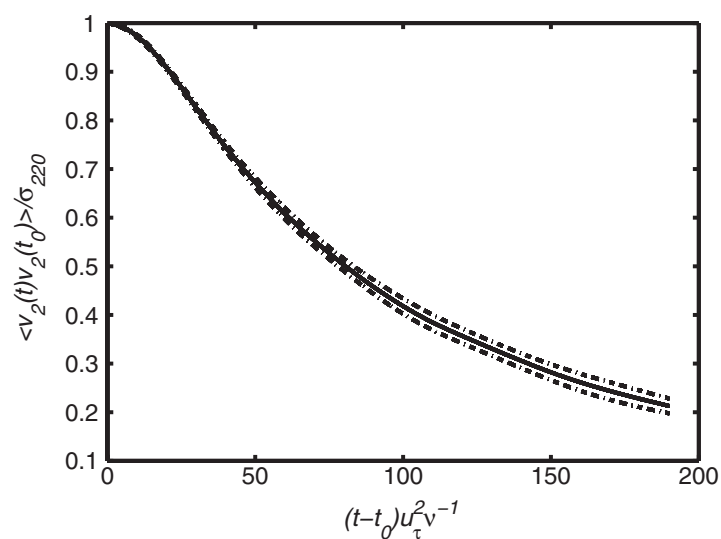

FIG. 2. Lagrangian auto-correlations obtained from DNS (solid) together with the $2 \sigma$ error bounds (dashed) for $x_{20}=$ $550 v u_{\tau}^{-1}$.

$t-t_{0}$ and the wall-normal coordinate of the particle $x_{20}$ at initial time $t_{0}$. Hence, they are averaged over all particles which start at the same $x_{20}$ position. For $t=t_{0}$ the velocity correlation function should equal the Eulerian velocity variance. The Lagrangian results yield a value which is equal to the Eulerian average within the error bounds for all wall-normal positions.

From the 300 independent results the statistical error in the correlation functions can be estimated. A results is shown in Fig. 2, where the correlation function of the wall-normal velocity component is shown for $x_{20}=550 v u_{\tau}^{-1}$ as a function of time together with the $2 \sigma$ error bounds. This implies that there is a probability of $87 \%$ that the correlation function lies between the two dashed lines. The correlation function is normalized with the result for $t=t_{0}$. The figure shows that the number of independent results is sufficiently large.

Some of the particles that are initially close to one of the walls exhibit spiraling motion. For particles which start further away from the walls, at $x_{2} u_{\tau} v^{-1} \geq 100$, such spiralling motion has not been found. This is illustrated in Fig. 3, where the projection of the particle trajectory on a plane perpendicular to the streamwise direction is plotted for two typical cases, one close to the wall and one away from the wall. The left figure illustrates the typical structures that are present close to the walls in flows with strong shear.
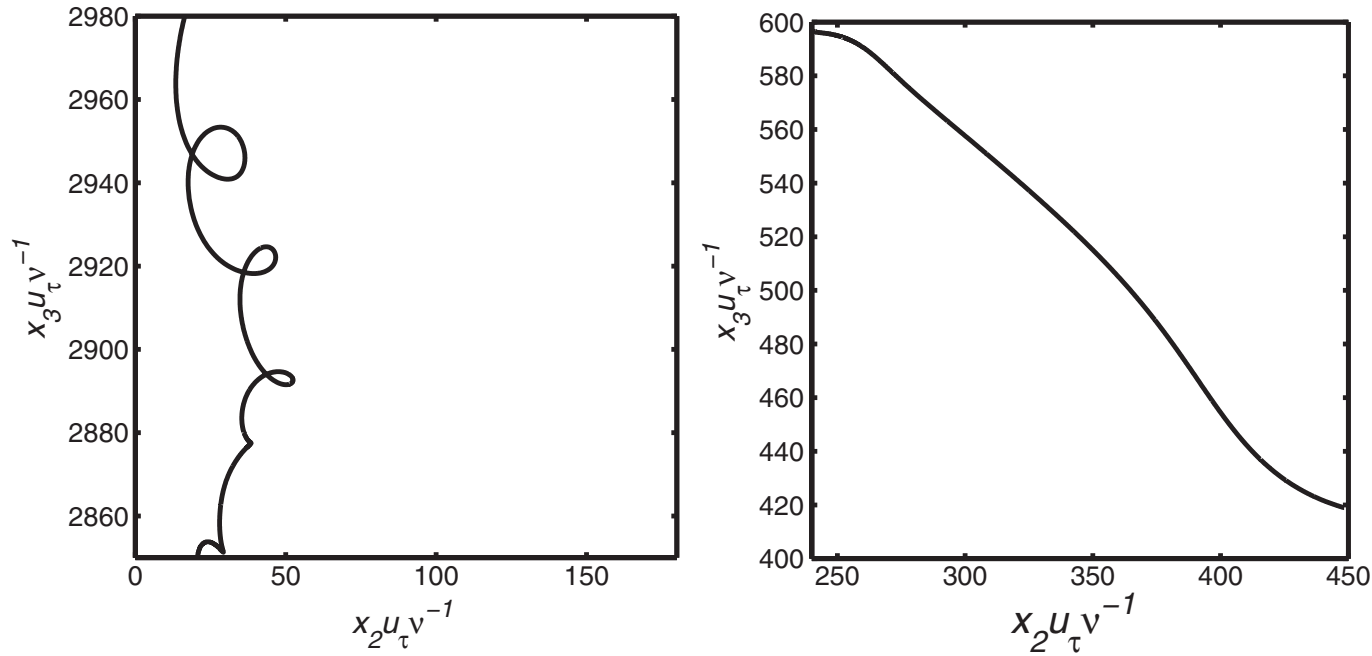

FIG. 3. Projection of particle trajectories on a plane perpendicular to the streamwise direction for two particles with initial wall-normal coordinate close to one of the walls (left) and not close to the walls (right). 


\section{LANGEVIN MODEL}

In this section and in Sec. IV we will describe the statistical models for dispersion of fluid particles that are compared in this paper with the DNS results. A well-defined method to model Lagrangian statistics is by a Langevin equation. The equation complies with the asymptotic structure of turbulence at large Reynolds number; its white noise term can be specified in accordance with the inertial subrange representation of Lagrangian Kolmogorov theory. ${ }^{4-6}$ The equation reads

$$
\frac{d v_{i}^{\prime}}{d t}=a_{i}^{\prime}\left(\mathbf{v}^{\prime}, \mathbf{x}\right)+\left\{C_{0} \epsilon(\mathbf{x})\right\}^{1 / 2} w_{i}(t), \quad \text { for } i=1,2,3,
$$

where $v_{i}^{\prime}=v_{i}^{\prime}(t)$ is the statistical representation of fluctuating fluid particle velocity relative to the Eulerian mean velocity $u_{i}^{0}(\mathbf{x})$ evaluated at particle position $\mathbf{x}=\mathbf{x}(t)$. Velocity is related to position by

$$
\frac{d x_{i}}{d t}=u_{i}^{0}(\mathbf{x})+v_{i}^{\prime} .
$$

In Eq. (5) $a_{i}^{\prime}\left(\mathbf{v}^{\prime}, \mathbf{x}\right)$ is the damping function, $C_{0}$ is the universal Kolmogorov constant, $\epsilon(\mathbf{x})$ is the mean energy dissipation rate at fixed position, and $w_{i}(t)$ is the Gaussian white noise of unit intensity. This implies that $\left\langle w_{i}(t)\right\rangle=0$ and $\left\langle w_{i}(t) w_{j}\left(t^{\prime}\right)\right\rangle=\delta\left(t-t^{\prime}\right) \delta_{i j}$ with $\delta_{i j}$ the Kronecker tensor, which is equal to 1 , if $i=j$ and zero otherwise. Gaussian white noise can be written as $w_{i}(t)=d W_{i}(t) / d t$, where $W_{i}(t)$ is a Wiener process. An expression for the damping function can be obtained by application of a perturbation expansion in which the reciprocal Kolmogorov constant serves as small parameter. ${ }^{7-9}$ Including terms of leading order and next-to-leading order with respect to $C_{0}^{-1}$ one has ${ }^{7,9}$

$$
a_{i}^{\prime}=-\frac{1}{2} C_{0} \lambda_{i j} \epsilon v_{j}^{\prime}+\frac{1}{2} \lambda_{j m} u_{k}^{0} \frac{\partial \sigma_{m i}}{\partial x_{k}} v_{j}^{\prime}+\frac{1}{2} \lambda_{j n} \frac{\partial \sigma_{i j}}{\partial x_{m}}\left(v_{m}^{\prime} v_{n}^{\prime}-\sigma_{m n}\right),
$$

where $\sigma_{i j}$ is the Eulerian co-variance or Reynolds stress tensor and $\lambda$ is its inverse.

The above result corresponds to an earlier proposal of Thomson. ${ }^{2,17}$ It was obtained under the assumption of a Gaussian probability density function for Eulerian (fixed-point) fluid velocity and satisfies the well-mixed criterion. ${ }^{2,17}$ However, the validity of this model has long been uncertain, because it was one of the many possible formulations of the damping function which satisfy the well-mixed criterion. ${ }^{2,6,9,17}$ The $C_{0}^{-1}$-expansion brought clarity to this issue, ${ }^{9}$ since it showed that the statistics of particle displacement obtained through application of the model according to Eq. (7) is unique up to a relative error of $C_{0}^{-2}$, see Brouwers. ${ }^{9}$ Investigation of the accuracy of this model using the results of DNS of channel flow is one of the objectives of the present work.

A drawback of the above Langevin model is that it cannot accurately describe the behavior of auto-correlation functions of velocity for very short correlation times. This is a consequence of the Markov model for velocity by which the smoothing of discontinuities by viscosity has been eliminated. The effect of finite viscosity on correlation functions becomes particularly noticeable at limited values of the Reynolds number. A customary way to model this effect is to apply the Markov approximation to acceleration rather than to velocity, ${ }^{5,9,18}$ a method also known as coloring the noise. ${ }^{19}$ We replace the white noise term in the Langevin equation by colored noise, according to

$$
\frac{d v_{i}^{\prime}}{d t}=a_{i}^{\prime}\left(\mathbf{v}^{\prime}, \mathbf{x}\right)+\left\{C_{0} \epsilon(\mathbf{x})\right\}^{1 / 2} f_{i}(t),
$$

where $f_{i}(t)$ in line with ordinary (K-41) Kolmogorov theory is described by the Langevin model:

$$
\frac{d f_{i}}{d t}=-\frac{f_{i}}{\tau_{\eta}}+\frac{\left(1+\eta_{i}\right)^{1 / 2}}{\tau_{\eta}} w_{i}(t)
$$

where $\tau_{\eta}=(v / \epsilon)^{1 / 2}$ is the Kolmogorov time and

$$
\eta_{i}=\frac{C_{0} \epsilon^{1 / 2} v^{1 / 2}}{2 \sigma_{i i}}
$$


is the ratio of the Kolmogorov time and the Lagrangian velocity correlation time. ${ }^{9}$ The statistical model according to (8) and (9) implies Gaussianity of $(d / d t) v_{i}^{\prime}(t)$. The effects of intermittency apparent in particle accelerations are thus not captured. Although important in acceleration, intermittency has only limited effect on the statistics of velocity and displacement. ${ }^{20}$ The above model is expected to yield appropriate corrections for finite viscosity on velocity correlations and displacement statistics. Its performance will be evaluated by a comparison with results of DNS.

Numerical solution of the Langevin equations should be treated with care, since the coefficients $\lambda_{i j}$ of the linear terms in $a_{i}^{\prime}$ in Eq. (7) diverge close to the walls of the channel. Therefore, in the numerical solution of Eqs. (6), (8), and (9) the exact solution of the linear system of equations with constant coefficients during each time step ${ }^{21}$ has been used. While the coefficients $u_{i}^{0}, \lambda_{i j}, \sigma_{i j}$, and $\epsilon$ are kept constant during one time step, they are adapted to their local, $x_{2}$-dependent value after each time step. These values correspond to the results obtained from the DNS, averaged over time and the two homogeneous directions at fixed wall-normal coordinate. The nonlinear terms are treated with the Euler forward method. The value of the time step is the same as in the DNS. Results are averaged over 100000 particles for each initial value $x_{20}$.

\section{HAMILTONIAN MODEL}

Apart from the Langevin model based on Eqs. (6), (8), and (9), the Lagrangian results of the DNS will be compared with those of a Hamiltonian stochastic model. The expression for the damping function according to Eq. (7) complies with the outcome of a two-term expansion with respect to $C_{0}^{-1}$. The first term of the expansion corresponds to a Hamiltonian base case. For large values of $C_{0}$ correlations of fluid particle velocity decrease in a short time. During this short time the change of energy due to dissipation will be small. The underlying mechanics of fluid particle velocity will be close to that of a Hamiltonian process. Analogous to the methods applied in statistical mechanics of molecular motion, one can impose Onsager symmetry. It results in a symmetrical linear damping term as described by the first term on the right-hand side of Eq. (7) with a truncation error of order $C_{0}^{-1}$ in statistical properties of particle velocity, but of order $C_{0}^{-2}$ in statistical properties of particle displacement. ${ }^{9}$ During the short times of correlation, the marked particle will have hardly moved so that a locally homogeneous field can be assumed to exist. The model according to the Hamiltonian base case can be described as

$$
\begin{gathered}
\frac{d v_{i}^{\prime}}{d t}=-\frac{1}{2} C_{0} \lambda_{i j 0} \epsilon_{0} v_{j}^{\prime}+\left(C_{0} \epsilon_{0}\right)^{1 / 2} f_{i}(t), \\
\frac{d f_{i}}{d t}=-\frac{f_{i}}{\tau_{\eta 0}}+\frac{\left(1+\eta_{i 0}\right)^{1 / 2}}{\tau_{\eta 0}} w_{i}(t),
\end{gathered}
$$

where the subscript 0 refers to values at the position and time where the particle starts. Equations for the correlation functions can be derived from Eqs. (11) and (12) as

$$
\begin{gathered}
\frac{d}{d t}\left\langle v_{n}^{\prime}(0) v_{i}^{\prime}(t)\right\rangle=-\frac{1}{2} C_{0} \lambda_{i j 0} \epsilon_{0}\left\langle v_{n}^{\prime}(0) v_{j}^{\prime}(t)\right\rangle+\left(C_{0} \epsilon_{0}\right)^{1 / 2}\left\langle v_{n}^{\prime}(0) f_{i}(t)\right\rangle, \\
\frac{d}{d t}\left\langle v_{n}^{\prime}(0) f_{i}(t)\right\rangle=-\frac{1}{\tau_{\eta 0}}\left\langle v_{n}^{\prime}(0) f_{i}(t)\right\rangle,
\end{gathered}
$$

which have been solved in closed form. ${ }^{9}$

\section{VELOCITY CORRELATIONS}

The results of the DNS have been used to calculate the three auto-correlations and crosscorrelations of fluid particle velocity. They have been compared with the results of time simulations of fluctuation Eqs. (6), (8), and (9). Values for the Eulerian quantities $u_{1}^{0}(\mathbf{x}), \epsilon(\mathbf{x})$, and $\sigma_{i j}(\mathbf{x})$ in these equations have been calculated from the DNS database. As mentioned above, they were adapted to their local values according to the DNS database when following a particle during the simulation. The Kolmogorov constant $C_{0}$ has been chosen equal to 6 . This was found to be the value which 
gives the best agreement with all DNS results. A third source for correlation functions is provided by the results obtained from the Hamiltonian base case, i.e., the solutions obtained in closed-form by dropping the nonlinear part in the damping term of the Langevin equation and keeping the space dependent parameters $\epsilon(\mathbf{x})$ and $\sigma_{i j}(\mathbf{x})$ constant and equal to their values at the point $x_{20}$ where they start. ${ }^{9}$ Also here $C_{0}=6$ has been chosen.

In Figs. 4 and 5, we have plotted the results for auto-correlations and cross-correlations, respectively, at four $x_{20}$ positions. The complete set of results for all $x_{20}$ positions is presented at http://www.tue.nl/ptc. Figures 4 and 5 show that the correlation functions according to the statistical model of Eqs. (6), (8), and (9), with locally adapted values of $u_{1}^{0}(\mathbf{x}), \epsilon(\mathbf{x})$, and $\sigma_{i j}(\mathbf{x})$, and those according to the Hamiltonian base model are almost the same for all $x_{20}$ positions. Only close to the wall some differences are observed, in particular, in the auto-correlation function of the wall-normal velocity component. The reason for the agreement is that displacement of a fluid particle in wall-normal direction is small during the time of correlation. ${ }^{9}$ Moreover, variations in the coefficients of the Langevin equation are partly canceled due to the displacement of a fluid particle in wall-normal direction. Close to the wall this cancellation effect is smaller, since the coefficients in the Langevin equation are more nonlinear there than in the center region of the channel. The results of the statistical models and those of DNS agree well at distances away from the wall, i.e., for $x_{20} \gtrsim 100 v u_{\tau}^{-1}$. For $u_{\tau}=1 \mathrm{~m} / \mathrm{s}$ and $v=10^{-5} \mathrm{~m}^{2} / \mathrm{s}$ this corresponds to approximately $1 \mathrm{~mm}$ and larger. In particular, the auto-correlation functions of the wall-normal and spanwise velocity components show good agreement, while differences are somewhat larger for the streamwise velocity component. The cross-correlations shown in Fig. 5 show to what extent the Onsager symmetry assumption is valid. Close to the walls the antisymmetric part of the cross-correlation found from the DNS is still quite large, whereas the Langevin models obviously predict zero. Further away from the wall the antisymmetric part of the cross-correlation obtained from the DNS gradually decreases to zero. For $x_{20}=896 v u_{\tau}^{-1}$ the agreement in the symmetric part of the cross-correlation seems less good, but note that the cross-correlation tends to zero in the center of the channel and the absolute difference between the DNS results and the results of the statistical models is still as small as closer to the walls.

For the correlation functions (see http://www.tue.nl/ptc) significant deviations between the results of DNS and of the statistical models occur in the near-wall region, $x_{20} \lesssim 100 v u_{\tau}^{-1}$. A likely reason is that for $x_{20} \lesssim 100 v u_{\tau}^{-1}$ the ratio of the time scales of the large scales and that of the small viscous scales $\eta_{i}^{-1}$ is no longer large, see Fig. 6 . The concept of separation of scales which underlies the statistical models is no longer valid. Yet, the deviation in diffusion coefficient is limited, as will be shown in Sec. VI. In practical dispersion modeling it may be advisable to introduce a near-wall region, where the wall conditions and those applied to the statistical model are connected by a wall function, a methodology known from Computational Fluid Dynamics (CFD). ${ }^{22}$

Figure 7 shows the variance of the wall-normal particle displacement, $\left\langle\Delta x_{2}^{2}\right\rangle-\left\langle\Delta x_{2}\right\rangle^{2}$ as a function of time for various initial positions $x_{20}$. The figure compares DNS results with the results of the statistical model based on Eqs. (6), (8), and (9). The agreement between the DNS results and the statistical model is good for initial particle positions not too close to the wall. Close to the center of the channel the agreement is also less good, in agreement with the results for the Lagrangian auto-correlations. While the statistical model underpredicts wall-normal dispersion close to the wall, it overpredicts this quantity close to the center of the channel.

\section{DIFFUSION COEFFICIENT}

Employing $C_{0}^{-1}$ as a small parameter it is possible to derive the diffusion equation as the "longtime-limit" of the Fokker-Planck equation associated with the Langevin model of Eqs. (5)-(7). Confining attention to turbulence in which Eulerian fixed-point statistical quantities are constant in the direction of the mean flow, the diffusion tensor in this equation is found to $\mathrm{be}^{7,9}$

$$
D_{i j}^{0}=2 C_{0}^{-1} \epsilon^{-1} \sigma_{i n} \sigma_{n j},
$$

where $\epsilon$ and $\sigma$ are dependent on the wall-normal coordinate. The diffusion tensor equals the time integral of the velocity correlation functions. ${ }^{1,9}$ This opens the possibility to extend expression (15) 

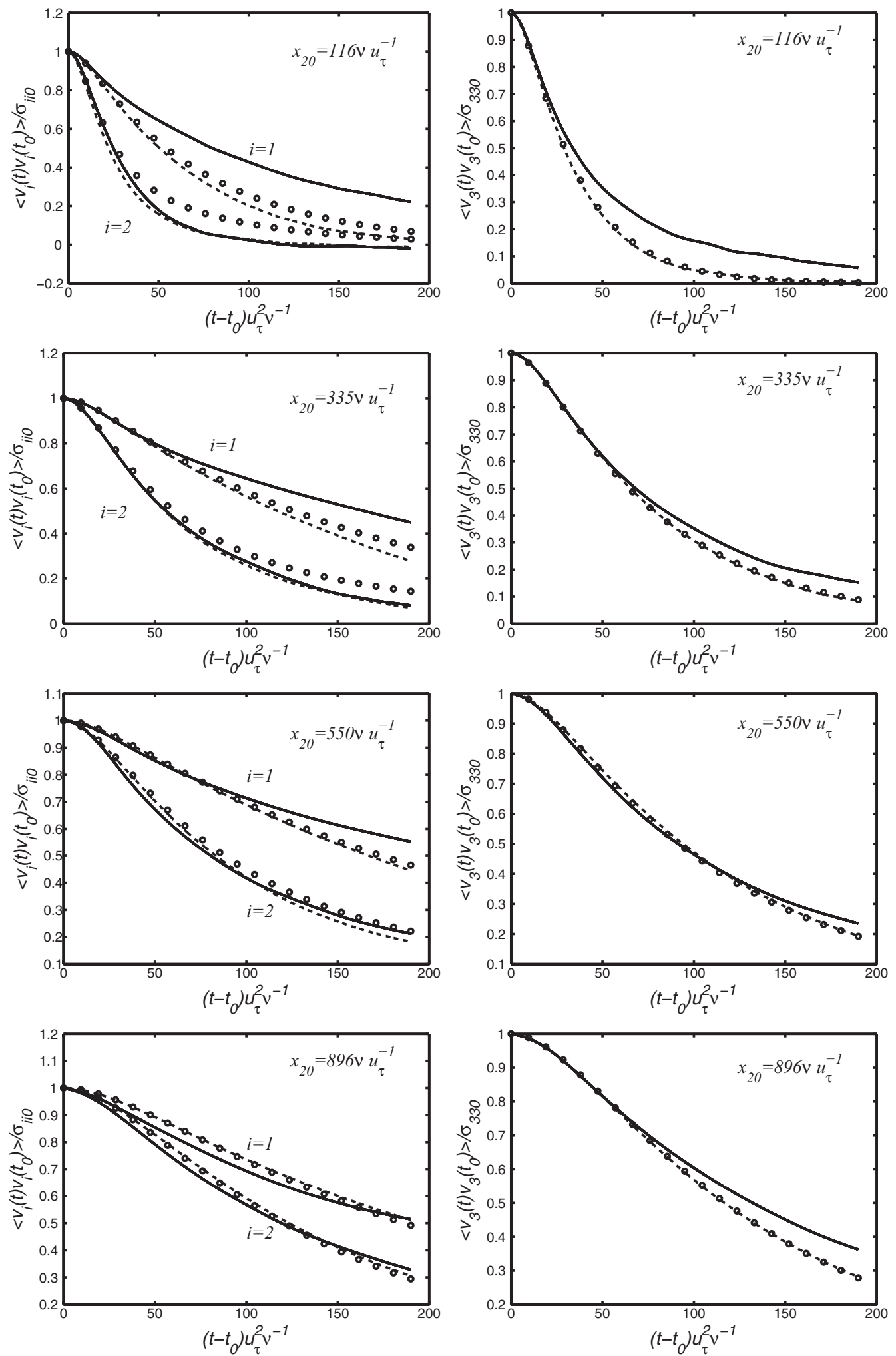

FIG. 4. Lagrangian auto-correlations obtained from DNS (solid), the statistical model of Eqs. (6), (8), and (9) (dashed), and the Hamiltonian base model (circles). 

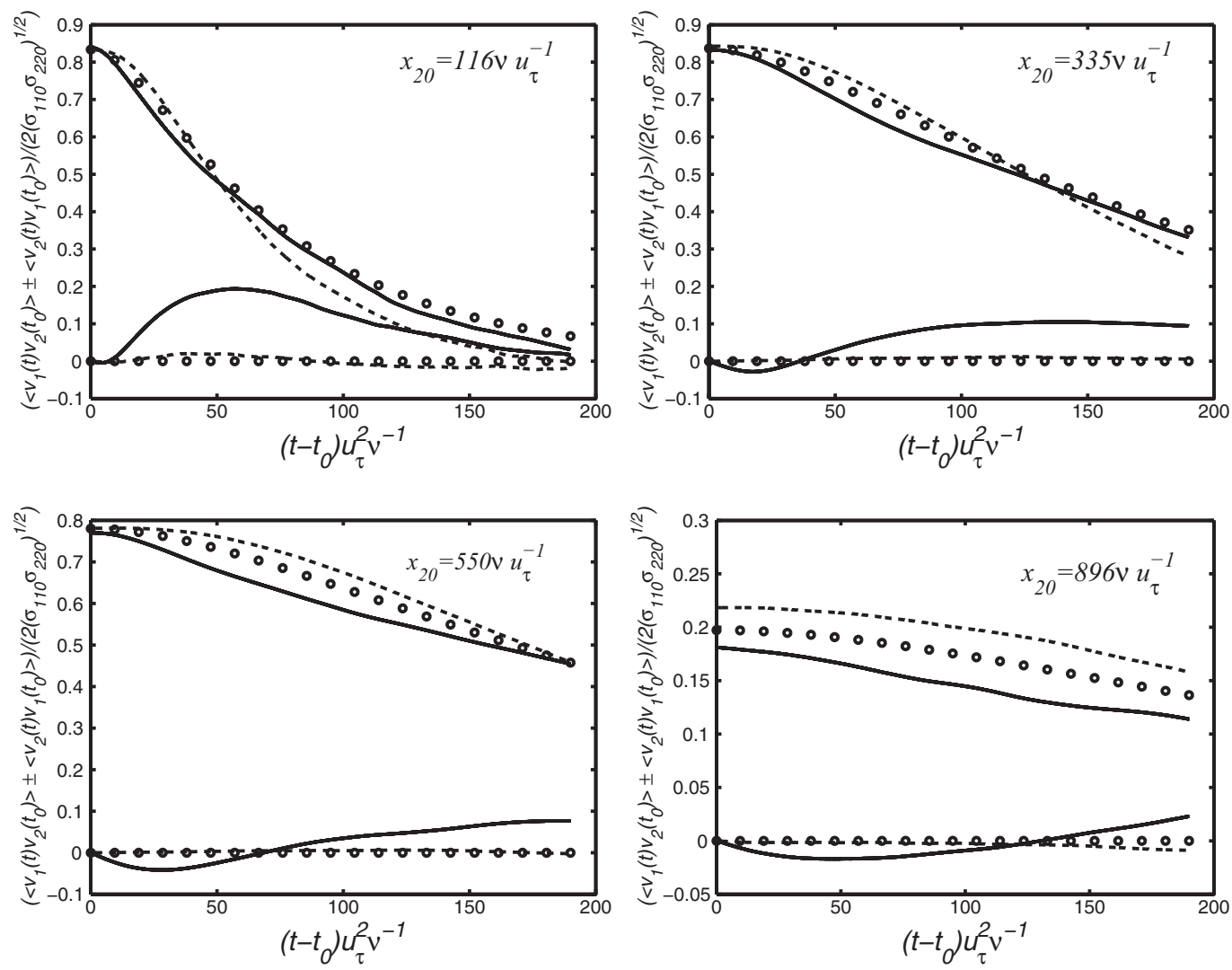

FIG. 5. Lagrangian cross-correlations of longitudinal and wall-normal velocities (symmetrical and anti-symmetrical parts) obtained from DNS (solid), the statistical model of Eqs. (6), (8), and (9) (dashed), and the Hamiltonian base model (circles).

to the case of finite viscosity, that is, by applying the previously derived expressions for correlations which include the effect of viscosity. For the diffusion coefficient one then finds

$$
D_{i j}= \begin{cases}D_{i i}^{0}\left(1+\eta_{i}\right) & \text { for } i=j \\ D_{i j}^{0} & \text { for } i \neq j,\end{cases}
$$

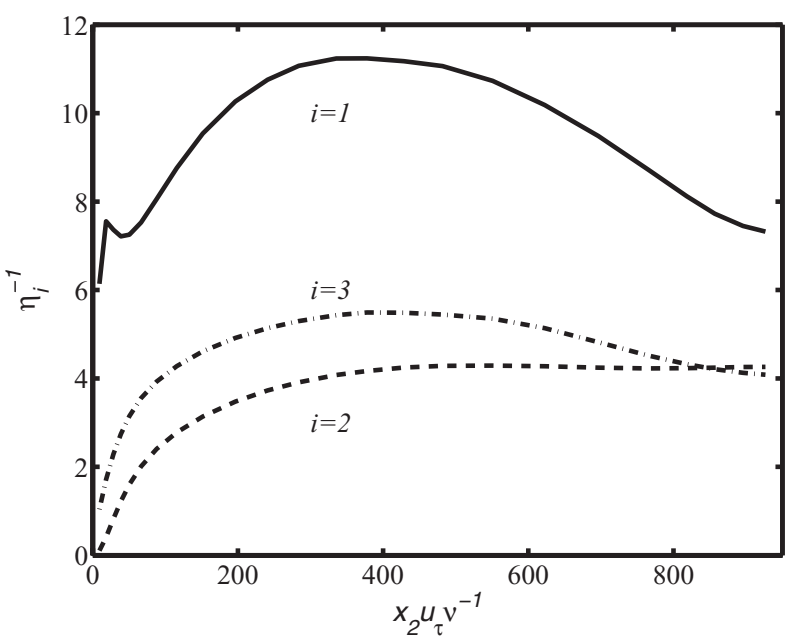

FIG. 6. Ratio of Lagrangian velocity time scale and Kolmogorov time as a function of distance from the wall. 

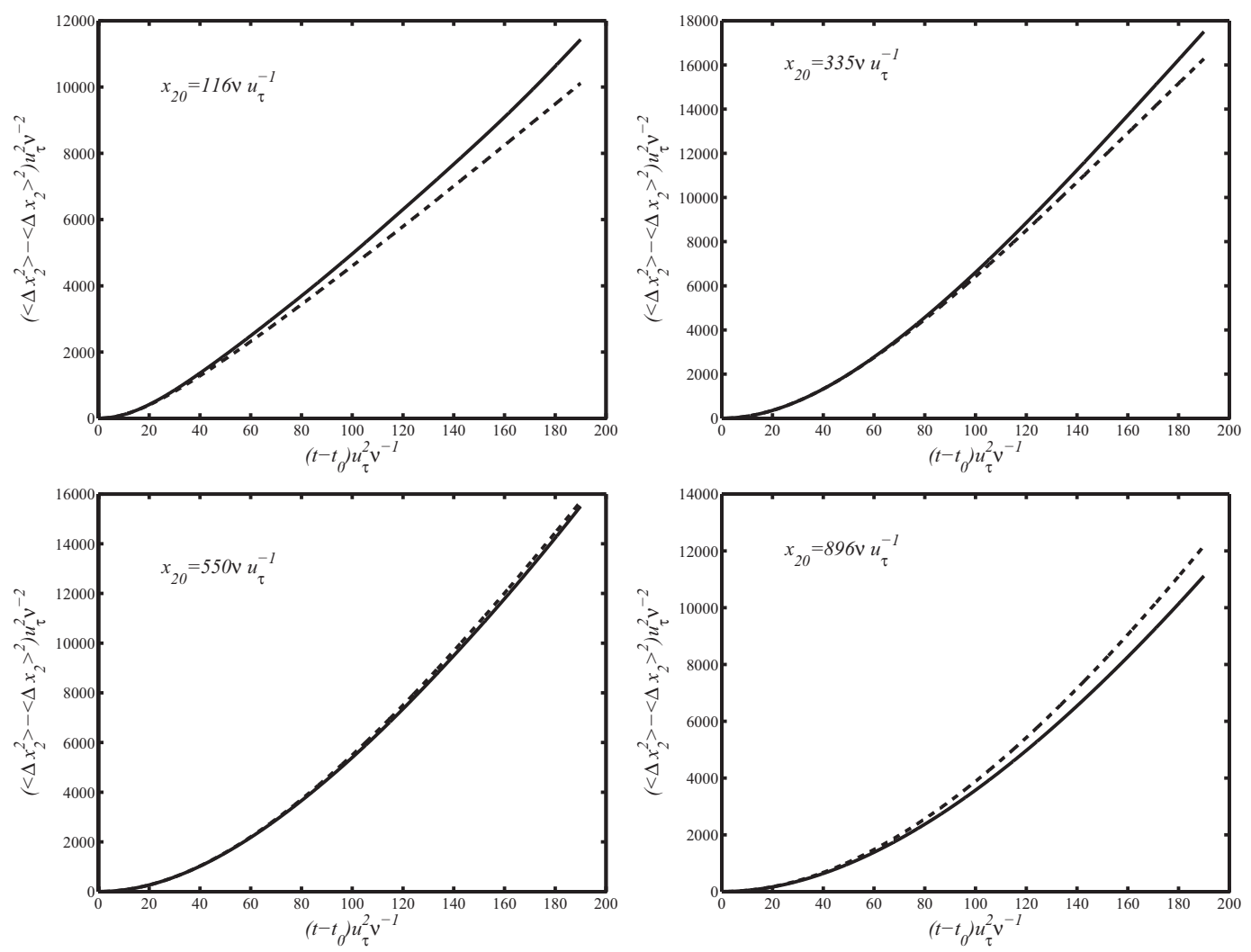

FIG. 7. Variance of particle displacement in wall-normal direction obtained from DNS (solid) and the statistical model of Eqs. (6), (8), and (9) (dashed).

with an error of $\mathcal{O}\left(\eta_{i}^{2}\right)$. This result quantifies how the diagonal components of the turbulent diffusion coefficient approach the inviscid limit as $\eta_{i} \rightarrow 0$.

At the present Reynolds number the diffusion limit is reached ${ }^{8}$ for $t u_{\tau}^{2} v^{-1} \approx 4500$, which is much longer than the time during which we track passive particles in the DNS $\left(t u_{\tau}^{2} v^{-1}=190\right)$. Therefore, it is not meaningful to compare the particle dispersion results obtained from the DNS with results of diffusion theory. Instead, we follow a different procedure to assess turbulent diffusion from the DNS results.

By adding an equation for a passive temperature to the DNS and imposing a temperature difference over the wall-normal direction, a fluctuating temperature field $\theta(\mathbf{x}, t)$ has been computed. ${ }^{16}$

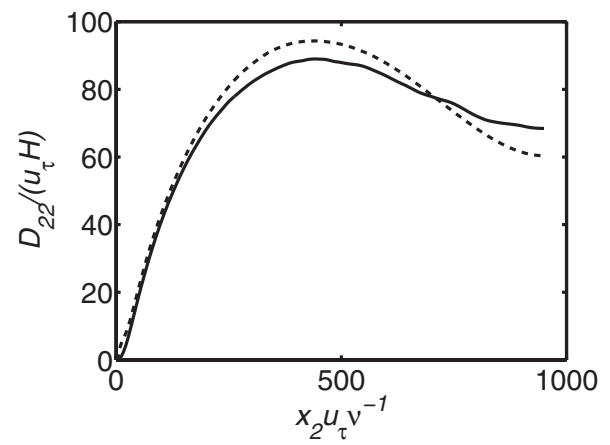

FIG. 8. Distribution of turbulent wall-normal diffusion coefficient calculated by DNS (solid line) and from theoretical expression (16) (dashed). 


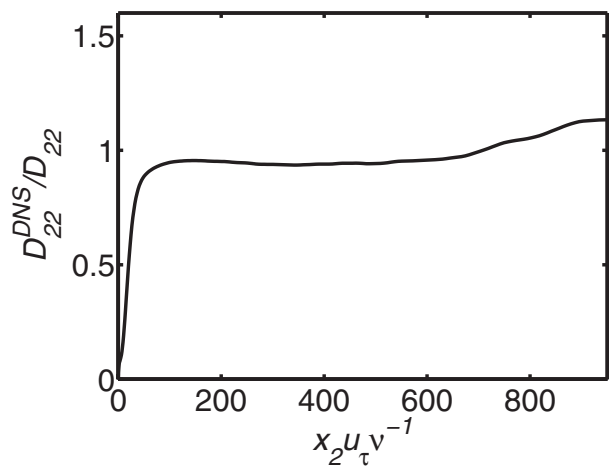

FIG. 9. Distribution of turbulent wall-normal diffusion coefficient calculated by DNS and divided by $D_{22}$ (solid) according to Eq. (16).

This enables the calculation of the wall-normal diffusion coefficient from the DNS results according to the relation

$$
D_{22}^{D N S}\left(x_{2}\right)=-\frac{\left\langle u_{2}^{\prime}(\mathbf{x}, t) \theta(\mathbf{x}, t)\right\rangle}{d\langle\theta(\mathbf{x}, t)\rangle / d x_{2}},
$$

where the brackets denote averaging over the homogeneous directions and time at the fixed wallnormal coordinate $x_{2}$ and where $u_{2}^{\prime}(\mathbf{x}, t)$ is the fluctuating wall-normal Eulerian fluid velocity component.

In Fig. 8, it can be seen that the wall-normal diffusion coefficient obtained from DNS agrees reasonably well with the result obtained from the statistical model according to Eq. (16), in which the effect of finite viscosity is taken into account. Results from the DNS divided by the theoretical values according to Eq. (15) versus $x_{2}$ are shown in Fig. 9. While the relative deviation is limited away from the wall, it becomes large in the near-wall region.

\section{CONCLUSIONS}

In this paper for the first time Lagrangian statistical results of turbulent channel flow at $\operatorname{Re}_{\tau}=$ 950 calculated with DNS have been presented. In particular, correlation functions of fluid particle velocity and results for the wall-normal diffusivity have been considered. As long as the distance to the wall is sufficiently large, the results compare well with predictions based on recently developed statistical models, which are based on Kolmogorov theory and Onsager symmetry. For smaller distances to the wall the viscous effects are too large to apply Kolmogorov theory.

Deviations between the results may have three causes: (1) The Reynolds number of the DNS is still limited, which leads to a limited separation between small and large scales. This effect has partly been corrected by the implementation of a higher order statistical model, which includes the Lagrangian acceleration, but this is not more than a useful approximation. (2) The assumption of Onsager symmetry is valid up to $\mathcal{O}\left(C_{0}^{-1}\right)$ in the correlation functions, which results in errors of $\mathcal{O}\left(C_{0}^{-2}\right)$ in particle displacement. (3) Intermittency of the turbulence is not included in Kolmogorov theory. The spiraling behavior of some particle tracks close to the walls, which is illustrated in Fig. 3, has no influence on the validity of the statistical models. The reason is that such particle tracks only occur close to the walls, where the statistical models are not valid since the ratio of the time scales of the large scales and that of the viscous scales is not large.

It has been shown that reduction of the Langevin model with coefficients which depend on the wall-normal coordinate to a Hamiltonian model with constant coefficients hardly changes the results. Only close to the wall, where the inhomogeneity of the turbulence is the largest, small changes can be observed, in particular, in the correlation function of the wall-normal velocity component.

A value for $C_{0}$ of about 6 was found to yield optimum agreement between the results of DNS and those of the statistical models. This holds for auto-correlations in all three directions, for the crosscorrelation, and for the wall-normal diffusion constant provided the correction for finite viscosity is 
taken into account. This conclusion is valid for all wall-normal distances away from the near-wall region. A value of $C_{0}$ of about 6 is somewhat less than the value recently mentioned in DNS-based studies of forced homogeneous isotropic turbulence. ${ }^{23}$

\section{ACKNOWLEDGMENTS}

This work was sponsored by the Stichting Nationale Computerfaciliteiten (National Computing Facilities Foundation, NCF) for the use of supercomputer facilities, with financial support from the Nederlandse Organisatie voor Wetenschappelijk Onderzoek, NWO. We thank the DEISA Consortium (www.deisa.eu), co-funded through the EU FP6 project RI-031513 and the FP7 project RI-222919, for support within the DEISA Extreme Computing Initiative.

${ }^{1}$ A. S. Monin and A. M. Yaglov, Statistical Fluid Mechanics, Mechanics of Turbulence (Dover, New York, 2007), Vol. I.

${ }^{2}$ D. J. Thomson, "Criteria for the selection of stochastic models of particle trajectories in turbulent flow," J. Fluid Mech. 180, 529 (1987).

${ }^{3}$ J. J. H. Brouwers, “On diffusion theory in turbulence,” J. Eng. Math. 44, 277 (2002).

${ }^{4}$ B. L. Sawford, "Recent development in the Lagrangian stochastic theory of turbulent dispersion," Boundary-Layer Meteorol. 62, 197 (1993).

${ }^{5}$ S. B. Pope, Turbulent Flows (Cambridge University Press, Cambridge, UK, 2000).

${ }^{6}$ J. D. Wilson and B. L. Sawford, "Review of Lagrangian stochastic models for trajectories in the turbulent atmosphere," Boundary-Layer Meteorol. 78, 191 (1996).

${ }^{7}$ J. J. H. Brouwers, "Langevin and diffusion equation of turbulent fluid flow," Phys. Fluids 22, 085102 (2010).

${ }^{8}$ J. J. H. Brouwers, "Langevin equation of a fluid particle in wall-induced turbulence," Theor. Math. Phys. 163, 677 (2010) [Teor. Mat. Fiz. 163, 328 (2010) (in Russian)].

${ }^{9}$ J. J. H. Brouwers, "Statistical description of turbulent dispersion," Phys. Rev. E 86, 066309 (2012).

${ }^{10}$ G. Falkovich, H. Xu, A. Pumir, E. Bodenschatz, L. Biferale, G. Boffetta, A. S. Lanotte, and F. Toschi, "On Lagrangian single-particle statistics," Phys. Fluids 24, 055102 (2012).

${ }^{11}$ S. Hoyas and J. Jiménez, "Scaling of velocity fluctuations in turbulent channels up to $R e_{\tau}=2000$," Phys. Fluids 18, 011702 (2006).

${ }^{12}$ B. J. Geurts and J. G. M. Kuerten, "Ideal stochastic forcing for the motion of particles in large-eddy simulation extracted from direct numerical simulation of turbulent channel flow," Phys. Fluids 24, 081702 (2012).

${ }^{13}$ J. Kim, P. Moin, and R. Moser, "Turbulence statistics in fully developed channel flow at low Reynolds number," J. Fluid Mech. 177, 133 (1987).

${ }^{14}$ P. R. Spalart, R. D. Moser, and M. M. Rogers, "Spectral methods for the Navier-Stokes equations with one infinite and two periodic directions," J. Comput. Phys. 96, 297 (1991).

${ }^{15} \mathrm{~S}$. Hoyas and J. Jiménez, "Reynolds number effects on the Reynolds-stress budgets in turbulent channels," Phys. Fluids 20, 101511 (2008).

${ }^{16}$ J. G. M. Kuerten, C. W. M. van der Geld, and B. J. Geurts, "Turbulence modification and heat transfer enhancement by inertial particles in turbulent channel flow," Phys. Fluids 23, 123301 (2011).

${ }^{17}$ B. L. Sawford and P. K. Yeung, "Lagrangian statistics in uniform shear flow: Direct numerical simulation and Lagrangian stochastic models," Phys. Fluids 13, 2627 (2001).

${ }^{18}$ B. L. Sawford, "Reynolds number effects in Lagrangian stochastic models of turbulent dispersion," Phys. Fluids A 3, 1577 (1991).

${ }^{19}$ N. G. van Kampen, Stochastic Processes in Physics and Chemistry, revised and enlarged ed. (Elsevier, Amsterdam, 1997).

${ }^{20}$ M. S. Borgas, "The multifractal Lagrangian nature of turbulence," Philos. Trans. R. Soc. London, Ser. A 342, 379 (1993).

${ }^{21}$ B. V. Ramarao, C. Tien, and S. Mohan, "Calculation of single fiber efficiencies for interception and impaction with superposed Brownian motion,” J. Aerosol Sci. 25, 295 (1994).

${ }^{22} \mathrm{~K}$. Hanjalić and B. Launder, Modelling Turbulence in Engineering and the Environment (Cambridge University Press, Cambridge, UK, 2011).

${ }^{23}$ B. L. Sawford and P. K. Yeung, "Kolmogorov similarity scaling for one-particle Lagrangian statistics," Phys. Fluids 23 , 091704 (2011). 